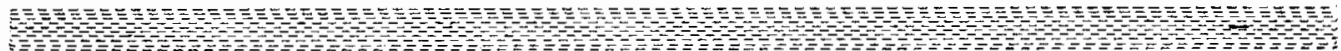

\title{
RESEARGH ARTICLES
}

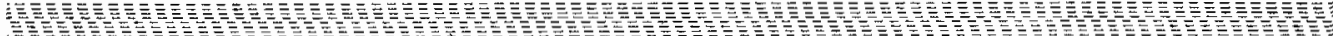

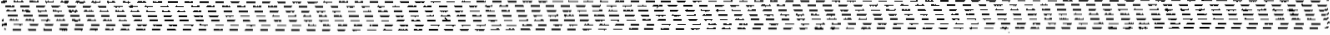

J. Sci. Soc. Thailand, 1 (1975), 220-225

\section{VALIDITY OF THE “GOLDEN RULE” FOR N-I-S TUNNELING}

\author{
RASSMIDARA NAVANI AND I-MING TANG
}

Department of Physics, Faculty of Science, Mahidol University, Rama VI Road, Bangkok, Thailand.

(Received 21 May 1975)

(Revised manuscript received 4 September 1975)

\section{Summary}

The transmission coefficients for forward and backward tunneling in a normal metal-insulator-superconductor (NIS) junction are obtained via the Bogoliubov-de Gennes equations. The fact that the two transmission coefficients are different casts doubt on the validity of the "golden rule" used in the transfer hamiltonian approach to tunneling in NIS junctions.

The transfer-hamiltonian formalism for the study of tunneling between a normal metal electrode and a superconducting electrode across an insulating barrier was first suggested by Bardeen 1 . In this approach to tunneling, the transfer hamiltonian

$$
\mathrm{H}_{\mathrm{T}}=\sum_{k, \mathrm{k}^{\prime}} \mathrm{V}_{\mathrm{Bk}, L \mathrm{k}^{\prime}} \mathrm{a}_{\mathrm{Rk}}+\mathrm{a}_{L k^{\prime}}+\mathrm{h} . \mathrm{c} .
$$

(where $\mathrm{R}$ and $\mathrm{L}$ refer to the electronic or quasiparticle states of the right hand side and the left hand side electrodes, respectively) is treated as a perturbation. To obtain the tunneling rate, one makes the crucial assumption that the transition rate for an electron in an initial (Block) state $\mathrm{k}^{\prime}$ of the normal electrode tunneling into a final quasiparticle state $\mathrm{k}$ of the superconducting electrode is given by the "golden rule"2 


$$
P_{f i}=\left(\frac{2 \pi}{\hbar}\right)\left|M_{f i}\right|^{2} \delta\left(\varepsilon_{k^{\prime}}-E_{k}\right)
$$

where $\mathrm{M}_{\mathrm{f}}^{\mathrm{z}}$ is the matrix element $\left\langle\mathrm{f}\left|\mathrm{H}_{\mathrm{T}}\right| \mathrm{i}>\right.$. With the transfer hamiltonian in the above form (equation 1), the transition rate for the reverse tunneling is also given by the above golden rule. It then follows that the net transition rate ${ }^{3}$ is given by

$$
P=\frac{2 \pi}{\hbar} \quad \int_{-\infty}^{\infty}|v(\omega)|^{2}\left\{f_{L}(\omega)-f_{R}(\omega)\right\} \rho_{L}(\omega) \rho_{R}(\omega) d \omega
$$

where $R(L)$ refers here to the right (left) hand side electrode; $f(\omega)$ is the Fermi-Dirac function; and $\rho(\omega)$ is the density of states.

The purpose of this paper is to show that the above golden rule is not valid for tunneling between a normal metal electrode and a superconducting electrode by using an alternative method, the Bogoliubov-de Gennes equation approach, 4,5 to obtain the net tunneling current. Recent investigations of the tunneling problem using a third technique, the Green's function method, 2,6 indicate that there are some shortcomings in the transfer hamiltonian approach. The transition rate obtained by the Green's function formalism contains an additional term called the "resonant term" since it contains an explicit dependence on the impurity spectral density in the barrier. This term exhibits a left-right asymmetry which is not present in the transfer hamiltonian transition rate. These studies do not, however, address themselves to the question of whether the golden rule is valid or not for tunneling between a normal metal electrode and a superconducting electrode.

Reason for doubting the validity of the golden rule is that in viewing the tunneling currents in the Bogoliubov-de Gennes equation approach, one sees an asymmetry in the current flows arising in the tunneling of the electrons from the normal metal side and in the tunneling of quasiparticles from the superconducting side. For tunneling from the normal metal side, we have an incident electron of momentum $k_{+}\left(k_{ \pm}=\left(k_{F}{ }^{2}-k_{13}{ }^{2} \pm\right.\right.$ $\left.2 \mathrm{mE} / \mathrm{h}^{2}\right)^{1 / 2}$ ) propagating towards the right, a reflected electron of momentum $k_{+}$and a hole of momentum -k_propagating towards the left, i.e.,

$$
\psi_{N}=\left\{\left(\begin{array}{l}
1 \\
0
\end{array}\right)\left[U_{+} e^{i k_{+} x}+u_{-} e^{-i k_{+} x}\right]+\left(\begin{array}{l}
0 \\
1
\end{array}\right) v_{-} e^{i k_{-} x}\right\} e^{\left.i k_{\|}\right|^{r} \|}
$$

where $k_{\|}$and $r_{\|}$are the components of the wave and position vectors parallel to the metalinsulator interface; and a transmitted electron-hole (BCS) pair propagating to the right in the superconducting region, i.e.,

$$
\psi_{S}=\left\{\left(\begin{array}{l}
1 \\
B
\end{array}\right) U_{+}^{+} e^{i k^{+} x}+\left(\begin{array}{l}
1 \\
B^{-}
\end{array}\right) U_{-}^{-} e^{-i k^{-} x}\right\} e^{i k}||^{x}||
$$




$$
\text { where } \quad \begin{aligned}
B^{ \pm} & =\Delta /(E \pm \Omega) \\
\Omega & =\left(E^{2}-\Delta^{2}\right)^{\frac{1}{2}} \\
\mathrm{k}^{ \pm} & =\left(\mathrm{k}_{\mathrm{F}}^{2}-\mathrm{k}_{\|}^{2} \pm 2 \mathrm{~m} \Omega / \hbar^{2}\right)^{\frac{1}{2}}
\end{aligned}
$$

For the case of tunneling from the superconducting side, we have in the superconductor an incident electron of momentum $\mathrm{k}^{+}$propagating towards the left and an electron-hole (BCS) pair propagating towards the right, i.e.,

$$
\psi_{\mathrm{B}}=\left\{U_{-}^{+}\left(\begin{array}{l}
1 \\
B
\end{array}\right) e^{-i k^{+} x}+U_{+}^{+}\left(\begin{array}{l}
1 \\
B
\end{array}\right) e^{i k^{+} x}+U_{-}^{-}\left(\begin{array}{c}
1 \\
B^{-}
\end{array}\right) e^{-i k^{-} x}\right\} e^{i k\left\|I_{1}\right\|}
$$

(where the last two wave functions represent the electron-hole pair) and in the normal metal, we have an electron of momentum $k_{+}$propagating towards the left and a hole of momentum- $\mathrm{k}_{\text {_ }}$ propagating towards the right, i.e.,

$$
\psi_{N}=\left\{U_{+}\left(\begin{array}{l}
1 \\
0
\end{array}\right) e^{-i k_{+} x}+v_{-}\left(\begin{array}{l}
0 \\
1
\end{array}\right) e^{i k_{-} x}\right\} e^{i k||^{x} \|}
$$

The transmission coefficient for both forward (from the normal side) and backward (from the superconducting side) tunneling is defined as $\mathrm{W}=\mathrm{J}$ transmitted/

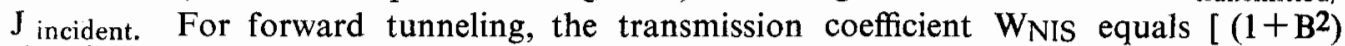
$\left.\left(\left|\mathrm{U}_{+}^{+}\right|^{2}-\mathrm{B}^{-2}\left|\mathrm{U}_{-}\right|^{2}\right)\right] /\left(\left|\mathrm{U}_{+}\right|^{2}+\left|\mathrm{V}_{-}\right|^{2}\right.$ where the $\mathrm{U}^{\prime} \mathrm{s}$ and $\mathrm{V}$ are the coefficients appearing in equations (4) and (5). For backward tunneling, the definition of the transmission coefficient leads to $\mathrm{W}_{\mathrm{SIN}}=\left(\left|\mathrm{U}_{+}\right|^{2}-\left|\mathrm{V}_{-}\right|^{2}\right) /\left[\left(1 \mathrm{~B}^{2}\right)\left(\left|\mathrm{U}_{ \pm}\right|^{2}+\mathrm{B}^{-2}\left|\mathrm{U}_{-}^{-}\right|^{2}\right)+4 \mathrm{Re} \mathrm{U}_{-}^{-*} \mathrm{U}_{ \pm}\right]$where the U's and $\mathrm{V}$ here are the coefficients appearing in equations (6) and (7). The coefficients appearing in equations (4)-(7) are determined by matching the wave functions (and their derivatives) for the normal metal or for the superconductor with the electronic wave functions in the insulator at the interfaces.

Since we are only interested in determining whether the golden rule is valid or not, we will consider in this study the simple but unphysical model of replacing the insulator by a rectangular potential barrier of height $\mathrm{V}_{\mathrm{O}}$. For such a model, the wave functions inside the insulator are

$$
\psi_{I}=\left\{\left(\begin{array}{c}
U_{+}^{I} \\
v_{+}^{I}
\end{array}\right) e^{k x}+\left(\begin{array}{c}
U_{-}^{I} \\
v_{-}^{I}
\end{array}\right) e^{-k x}\right\} e^{i k}\left\|^{r}\right\|
$$

where $\kappa=\left(k_{1}{ }^{2}+2 \mathrm{mV}_{\mathrm{o}} / \mathrm{h}^{2}\right) \frac{1}{2}$. Applying the boundary conditions at the interfaces, we obtain for both cases, eight equations with nine unknowns. Griffin and Demers 4 have 


$$
c_{n m}(t) \quad=\delta_{n m}+c_{n m}^{(1)}+c_{n m}^{(2)}+\ldots \ldots+c_{n m}(k)
$$

where $c_{n m}{ }^{(1)}$ is the coefficient determined in the first order perturbation theory; $c_{n m}{ }^{(2)}$, in the second order perturbation theory; and where $\mathrm{c}_{\mathrm{nm}}{ }^{(\mathrm{k})}$, in the $\mathrm{k}$-th order perturbation theory. With the transfer haniltonian (1) as the perturbation, the coefficients $c_{n m}(t)$ and $\mathrm{c}_{\mathrm{mn}}(\mathrm{t})$ can be written as

$$
c_{n m}(t)=\sum_{k, k} a_{n k}^{+} a_{m k}\left\{v_{n k, m k}{ }^{\prime}+\text { terms of order } v^{2}, v^{3}, \ldots, v^{k}\right\}
$$

and

$$
c_{m n}(t)=\sum_{k, k^{\prime}} a_{m k^{\prime}}{ }^{+} a_{n k}\left\{v_{m k}, n k^{+}+\text {terms of order } v^{+2} \ldots, v^{+2}\right\}
$$

The bracketed terms appearing in the two coefficients are not necessarily the complex conjugate of each other. Since the transfer hamiltonian (1) exhibits time reversal symmetry, we have by the principle of detailed balancing 7

$$
\mathrm{P}_{\mathrm{nm}}=\mathrm{P}_{\mathrm{mn}}
$$

i.e., the rate of transition for the state $n$ to go into a state $m$ is the same as the rate of transition for the state $m$ to go into a state $n$. It then follows from the definition of the transition rate that

$$
\left|c_{n m}(t)\right|^{2}=\left|c_{m n}(t)\right|^{2}
$$

This means that the modulus square of the bracketed term of eq. (15) is equal to the modulus square of the bracketed term of eq. (16) if the potential $\mathrm{V}$ does not depend strongly on $\mathrm{k}$ and $\mathrm{k}^{\prime}$ (this property of $\mathrm{V}_{\mathrm{nk}} \mathrm{mk}^{\prime}$ is usually assumed). As a consequence, the net current obtained in the higher order perturbation treatment of the transfer hamiltonian will be in the form given by (3) except that the $|\mathrm{V}(\omega)|^{2}$ factor is replaced by the modulus square of the bracketed term of eq. (15). Since the transfer hamiltonian approach is a phenomenological model with the potential $V$ to be determined, we can replace the potential appearing in eq. (1) with the modulus of the bracketed term of eq. (15). In this way, the net current obtained by the use of the golden rule will be the 'exact' net current.

The clue as to why the net current obtained by the Bogoliubov-de Gennes approach is different from that obtained by the transfer hamiltonian approach is provided by the fact that the principle of detailed balancing is based on time reversal symmetry. For NIS tunneling, there is no time reversal symmetry since the physical picture when the electrons are incident from the normal metal side is not the time reversal of the picture when the electrons are incident from the superconducting side. The transfer hamiltonian for NIS tunneling should be in the form 


$$
H_{T}=\sum_{k, k^{\prime}}\left\{v_{R k, L k^{\prime}}{ }^{(1)} a_{R k^{\prime}}^{\dagger} a_{L k^{\prime}}+v_{L k^{\prime}, R k}{ }^{(2)} a_{L k^{\prime}}{ }^{\dagger} a_{R k}\right\}
$$

where $\left(V_{R k}, L^{\prime}(1)+\neq V_{L^{\prime}}, R_{k}{ }^{(2)}\right.$. With this hamiltonian as the perturbation, the perturbative calculation of the net current would be in the form of the current obtained by the Bogoliubov-de Gennes approach. It is interesting to note that for superconductorinsulator-superconductor (SIS) tunneling ${ }^{8}$ where time reversal symmetry has been restored, the net current obtained by the Bogoliubov-de Gennes approach is in the form given by (3).

\section{References}

1. Bardeen, J. (1961) Phys. Rev. Lett. 6, 57.

2. The present wording of the "golden rule" is that of Feuchtwang, T.E. (1974) Phys. Rev. $B$ 10, 4121.

3. Appelbaum, J.A. and Brinkman, W.F. (1969) Phys. Rev. 186, 464.

4. Griffin, A. and Demers, J. (1971) Phys. Rev. B 4, 2202.

5. Navani, R. (1973) M.S. Thesis, Georgetown University.

6. Feuchtwang, T.E. (1974) Phys. Rev. B 10, 4135.

7. See for instance, Quantum Theory I. Elements (Bates, D.R., ed.,) Academic Press N.Y., (1961) Ch. 8, 251.

8. Navani, R. (1974) PhD. Thesis, Georgetown University.

\section{บทคัดย่อ}

ใน N-I-S junction ที่ประกอบด้วยโลหะธรรมดา $(\mathrm{N})$ ฉนวน (I) และ superconductor (S) การคำนวณหา transmission coefficients สำหรับ tunneling ในทิศจาก $\mathrm{N}$ ไปยัง $\mathbf{S}$ และ $\mathbf{S}$ ไปยัง $\mathbf{N}$ ทำได้โดยการใช้สมการ Bogoliubov-de Gennes จากการคำนวณ ได้ transmission coefficients ของกรณีทั้งสองไม่เท่ากัน ซึ่งเบ็นที่น่าคิดว่า ทฤษฎี “golden rule" จะนำมาใช้โดยตรงกับ transfer-hamiltonian ของ tunneling ใน N-1-S junction ไม่ได้ 


\section{THE EQUILIBRIUM CONDENSATE FRACTION IN SUPERFLUID HELIUM}

FREDERICK W. CUMMINGS

Department of Physics, University of California, Riverside, California, U.S.A. 92502

KITT VISOOTTIVISETH

Department of Physics, Khon Kaen University, Khon Kaen, Thailand.

(Received 20 October 1975)

Summary

This paper reports the temperature dependence of the condensate fraction in ${ }^{4} \mathrm{He} I \mathrm{I}$ determined from Cummings' proposal which involves only measurement of the liquid structure factor.

\section{Introduction}

It is widely believed that the ratio of the (bulk) condensate to the total density $\left(\rho_{c} / \rho\right)$ is in the range of 0.08 to 0.25 at $\mathrm{T}=\mathrm{O}^{\circ} \mathrm{K}$ for ${ }^{4} \mathrm{He}$ II, based on theoretical estimates ${ }^{1-4}$. An experimental confirmation of this theoretical estimates is feasible. One of us ${ }^{5}$ has presented a method for determining the condensate density fraction for all temperatures based only on the knowledge of the equilibrium pair distribution function $g(r, T)$ as a function of temperature just above $\mathrm{T}_{\lambda}$ and below.

\section{Theoretical Considerations}

We will brietly review first the method which has been proposed by Cummings 5 and then extend it. From the conditions ${ }^{6}$ required of the second order reduced density matrix $\Omega_{2}$ and the form ${ }^{6}$ for $\Omega_{2}$, we can write it as

$$
\begin{aligned}
\Omega_{2}(r) & =\rho^{2} g(r, T) \\
& =\phi_{1}^{2}(r) \rho_{c}^{2}+2 \delta_{2}^{2}(r) \rho_{c} \rho_{d}+2 \phi_{2}^{2}(r) \rho_{c}\left|A_{1}(r)\right|+\Lambda_{2}(r)
\end{aligned}
$$

which is valid when $r>r_{1} \simeq 4.5 \AA$ the point where the first order reduced density matrix 2 $\Omega_{1}(r)$ becomes equal to the condensate density $\rho_{c}$ (see fig. 1), and where $g$ assume the value 1 for the second time. The function $\Lambda_{2}$ must satisfy all conditions analogous to the conditions required of $\Omega_{2}$. Thus $\Lambda_{2}(0)=0$, and $\Lambda_{2}(r)=\rho_{d}^{2}$ when $r>r_{2}$, where $r_{2}$ is several times larger than $r_{1}$. Therefore $\left|\Lambda_{2}(r)\right|$ may be defined as

$$
\left|\Lambda_{2}(r)\right|=\rho_{\mathrm{d}}^{2} \tilde{g}(r, T)
$$

